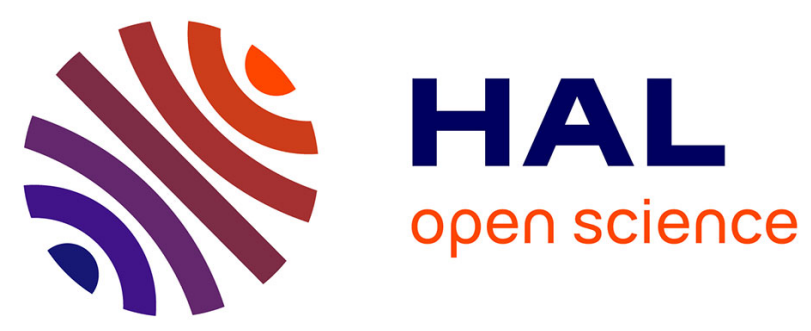

\title{
Velocities of speckles in a smoothed laser beam propagating in a plasma
}

\author{
François Walraet, Guy Bonnaud, Gilles Riazuelo
}

\section{To cite this version:}

François Walraet, Guy Bonnaud, Gilles Riazuelo. Velocities of speckles in a smoothed laser beam propagating in a plasma. Physics of Plasmas, 2001, 8, pp.4717 - 4720. 10.1063/1.1405128 . cea01709026

\section{HAL Id: cea-01709026 https://hal-cea.archives-ouvertes.fr/cea-01709026}

Submitted on 14 Feb 2018

HAL is a multi-disciplinary open access archive for the deposit and dissemination of scientific research documents, whether they are published or not. The documents may come from teaching and research institutions in France or abroad, or from public or private research centers.
L'archive ouverte pluridisciplinaire HAL, est destinée au dépôt et à la diffusion de documents scientifiques de niveau recherche, publiés ou non, émanant des établissements d'enseignement et de recherche français ou étrangers, des laboratoires publics ou privés. 
Velocities of speckles in a smoothed laser beam propagating in a plasma

François Walraet, Guy Bonnaud, and Gilles Riazuelo

Citation: Physics of Plasmas 8, 4717 (2001);

View online: https://doi.org/10.1063/1.1405128

View Table of Contents: http://aip.scitation.org/toc/php/8/11

Published by the American Institute of Physics

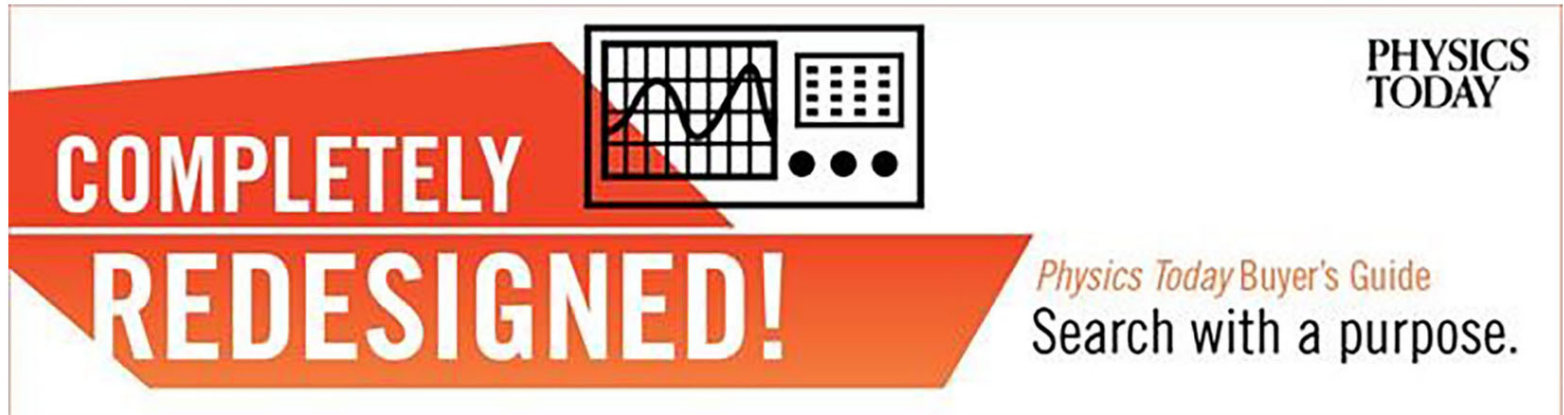




\title{
Velocities of speckles in a smoothed laser beam propagating in a plasma
}

\author{
François Walraet, Guy Bonnaud, ${ }^{\text {a) }}$ and Gilles Riazuelo \\ Commissariat à l'Energie Atomique, DPTA, BP 12, 91680 Bruyères-le-Châtel, France
}

(Received 12 April 2001; accepted 1 August 2001)

\begin{abstract}
By means of three-dimensional simulations, the paraxial propagation of a laser beam in a nonlinear plasma modeled by ion-acoustic waves is examined. The beam speckles are smoothed by a one-dimensional transverse spectral dispersion technique. In the case of weak smoothing and when the incident speckle velocity is subsonic, the propagation in the plasma is found to exhibit an asymptotic speckle velocity and to enhance filamentation. With contrary conditions, it is found to reduce simply the speckle velocity. (C) 2001 American Institute of Physics.
\end{abstract}

[DOI: $10.1063 / 1.1405128]$

In the context of laser-driven inertial confinement fusion, interest in filamentation stems from concerns over the potential of intensified speckles in the laser beam to dig density depressions in the plasma, which can refract the light rays, counter their natural diffraction, and induce self-focusing. The beam intensity distribution is thereby shifted to higher values, causing the onset of parametric instabilities leading to some fraction of the incident light to be scattered. ${ }^{1}$ In gas-filled hohlraum targets, as in experiments with the Nova laser and future megajoule lasers, ${ }^{2}$ the homogeneous transparent plasma, i.e., with electron density less than the critical density for laser reflection $\left(n_{c}=10^{21} / \lambda_{0}^{2} \mathrm{~cm}^{-3}\right.$ with $\lambda_{0}$ the laser wavelength in $\mu \mathrm{m}$ ), is a few millimeters long and can cause beam deviation and spreading, if the plasma expands transversely. ${ }^{3}$ This anomalous nonlinear speckle refraction is intended to be controlled via beam smoothing techniques, which will shorten the speckle size and lifetime and make the speckle scintillate rapidly. ${ }^{4}$ The smoothing by spectral dispersion (SSD) pioneered at Rochester ${ }^{4}$ has rapidly developed into a standard technique for the baseline of the present big laser projects.

During the past decade, the escalation in computer speed/memory made three-dimensional (3-D) simulations possible with realistic distances over tenths of picoseconds which are able to provide both the optics engineers and the target designers with helpful macroscopic quantities. Once the analytical scaling laws of filamentation were stated ${ }^{5}$ and identified by simulations, ${ }^{6}$ the impact of the SSD was estimated $^{7,8}$ as well as the influence of the plasma in terms of self-smoothing. ${ }^{9-11}$ To provide a new vision of selfsmoothing, previously studied in terms of speckle size and lifetime, ${ }^{10}$ this letter concentrates on the velocity of the speckles and reports on its variation as a function of the transverse SSD control parameters and on the possible resonance between this velocity and the plasma density-wave velocity.

Our discussion is based on simulation results provided by the code PARAX. ${ }^{10}$ We shall denote by $z, x, y$, and $t$, the main propagation axis, the two axes transverse to the $z$ axis,

aElectronic mail: bonnaud@bruyeres.cea.fr and the time, respectively. Periodic conditions are assumed in the $x-y$ plane. The propagation of one fixed polarization electromagnetic wave is modeled by one generalized scalar paraxial equation for the electric field amplitude $E .^{12}$ No absorption is considered and consequently propagation preserves the beam power. The prime mover of the plasma perturbation is, on the one hand, the laser ponderomotive force (PF, acting on electrons) which induces, on long time scales, the joint motion of electrons and ions and, on the other hand, the thermal force dominated by the electrons which are hotter than ions. The plasma density is modeled by using a fluid framework, where expansion to second order in the field perturbation leads to an ion-acoustic wave (IAW) driven by the PF. IAWs propagate only in the $x, y$ plane, according to Ref. 10 and Brillouin backscattering does not arise due to the paraxial concept of the code PARAX. In present plasma/laser conditions, filamentation and forward Brillouin scattering (FSBS) can grow and interact. ${ }^{9}$ FSBS has been diagnosed in our simulations via the frequency spectra of the $E$ field: they show bandwidth increase with distance, with noticeable extension on the red side very similar to Ref. 9 (where $x-z$ hydro response is involved). A full 3-D plasma response will be part of a future study to assess quantitatively the influence of the missing $z$ axis.

The plasma is assumed to be initially nondrifting and uniform with density $n_{e}(x, y, z, 0) \equiv n_{e 0}=0.1 n_{c}$. IAW damping is by collisionless Landau damping (valid for light ions). We have chosen the mass ratio $m_{i} / Z m_{e}=3672$, the electron temperature $T_{e}=3 \mathrm{keV}$, and the temperature ratio $T_{i} / Z T_{e}$ $=0.19$ ( $e$ and $i$ stand for the electrons and ions, respectively; $Z$ is the ion charge number), which lead to the IAW velocity (referred to the velocity $c$ of light in vacuum): $c_{s} / c=1.58$ $\times 10^{-3}$ and $\nu_{s} / \omega_{s}=0.1$ ( $\omega_{s}$ is the IAW angular frequency).

The electric field is imposed at the boundary $z=0$ such that a one-dimensional (1-D) SSD field pattern is generated inside the plasma. The SSD technique uses a sinusoidal phase modulator with frequency $\nu_{m}=10 \mathrm{GHz}$ and depth $\beta$ in the range [1-6], a grating with maximum delay time $N_{c} / \nu_{m}$ ( $N_{c}$ is defined as the number of color cycles), and a random phase plate (RPP with $2.4 \mathrm{~cm}$ step size) characterized by 0 and $\pi$ dephasing square steps. ${ }^{4}$ The laser wavelength is 1.053 $\mu \mathrm{m}$ up to the RPP and, after frequency tripling, becomes 
$\lambda_{0}=0.35 \mu \mathrm{m}$ in the focal spot, leading to the full laser bandwidth $\Delta \nu=6 \beta \nu_{m}$. The beam has a 36 -cm-side square shape and the focus number is $\#=8.33$. The average irradiance is $2 \times 10^{15} \mathrm{~W} / \mathrm{cm}^{2}$ which gives to a speckle whose maximal intensity is 8 times the average intensity a power equal to 1.43 times the critical power.

Herein, we concentrate on the mean velocity of the speckles in the irradiance pattern, calculated along both the transverse and axial directions. This velocity determines the coherence time $\tau_{c}$ of the speckle which can be very different from its lifetime $\tau_{l}$. The former characterizes the duration of one average light flash at a fixed point whereas the latter is the flash duration in the frame comoving at the speckle velocity. The speckle displacement can be quantified through the tranverse (respectively, axial) time $\tau_{\perp}$ (respectively, $\tau_{\|}$), defined as the time required for a speckle to cross one speckle width (respectively, length). For practical reasons, we define as coherence time $\tau_{c}=\min \left(\tau_{\perp}, \tau_{\|}, \tau_{l}\right)$.

We have calculated the correlation functions of the irradiance in the $(x, y)$ and $(x, z)$ planes:

$$
\begin{aligned}
C_{z, t, \delta t}(\delta x, \delta y)= & \iint d x d y I(x+\delta x, y+\delta y, z, t \\
& +\delta t) I(x, y, z, t), \\
C_{y, t, \delta t}(\delta x, \delta z)= & \iint d x d z I(x+\delta x, y, z+\delta z, t \\
& +\delta t) I(x, y, z, t)
\end{aligned}
$$

(via Fourier transform) and reported the values $\delta x=x^{*}$ and $\delta y=y^{*}$ (respectively, $\delta z=z^{*}$ ) such that $C$ maximizes, ${ }^{13}$ for given time $t$, time shift $\delta t$ and $z$ (respectively, $y$ ). The socalled speckle velocity, defined by $\left(v_{x}, v_{y}\right)$ $=\left(x^{*} / \delta t, y^{*} / \delta t\right)$, denotes the average velocity of the speckle pattern. We have chosen $\delta t$ to be the simulation time step (i.e., $0.125 \mathrm{ps}$ ).

Both transverse and axial velocities are complicated functions of time, $\nu_{m}, N_{c}$, the speckle width $w_{\text {sp }}$ and the length $l_{\text {sp }}$. By defining the modulation length $l_{m}$ $=c /\left(2 \beta \nu_{m}\right),{ }^{14}$ we can write $l_{\mathrm{sp}} / l_{m}=2 \pi \#^{2} \lambda_{0} \beta \nu_{m} / c=5$ $\times 10^{-4} \beta \nu_{m}$ with $\nu_{m}(\mathrm{GHz})$. As this ratio cannot exceed a few percent, the velocity has this simple sinusoidal time profile:

$$
\frac{v_{x}(t)}{c}=3 \frac{w_{\mathrm{sp}}}{l_{m}} C_{4} \sin \left(2 \pi v_{m} t\right)
$$

with $C_{4}=\sin \left(\pi N_{c}\right) /\left(\pi N_{c}\right)^{2}-\cos \left(\pi N_{c}\right) / \pi N_{c}$ (maximizing at $N_{c}=0.66$ ), according to Ref. 14 notations. For our conditions, the speckle velocity is proportional to $\beta$ and exhibits damped oscillations as a function of $N_{c}$. The axial velocity varies also sinusoidally in time:

$$
\frac{v_{z}(t)}{c}=\frac{45}{\pi} \frac{l_{\mathrm{sp}}}{l_{m}} C_{1} \cos \left(2 \pi \nu_{m} t\right)
$$

with

$C_{1}=\cos \left(\pi N_{c}\right) /\left(\pi N_{c}\right)^{2}+\sin \left(\pi N_{c}\right)\left[1 /\left(3 \pi N_{c}\right)-1 /\left(\pi N_{c}\right)^{3}\right]$.

Equation (4) is valid if $C_{1} \gg 10^{-3}$.

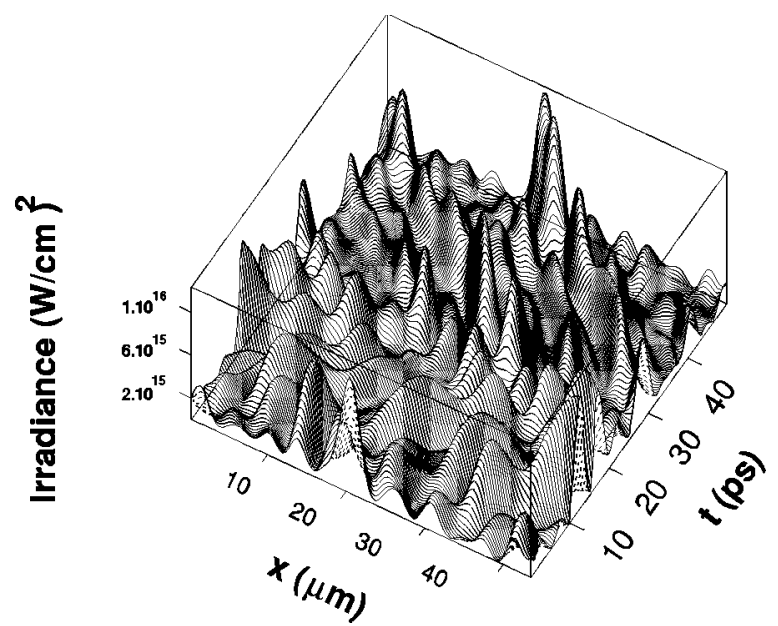

FIG. 1. Speckle pattern in the focal spot as a function of time. The laser parameters are: $\nu_{m}=10 \mathrm{GHz}, \beta=2, N_{c}=0.8$.

In Fig. 1, the set of speckles in $z=0$ provided by the code PARAX exhibits clearly a sinusoidal motion. The measured axial and transverse velocities are in full agreement with the velocities calculated by Eqs. (3) and (4): They have a nonmonotonic dependence on $N_{c}$ and scale linearly with $\beta$. Equation (3) exhibits ranges of $N_{c}$ values where $v_{x} / c_{s} \approx 1$, which are liable to trigger laser-plasma resonance effects, in contrast to the axial velocity which is most often one order of magnitude larger than $c_{s}$. For this reason, we will discuss mainly the results obtained for the transverse velocity. According to Ref. 14 , the ratio $\tau_{l} / \tau_{c}$ of the coherence time to the lifetime (averaged over one modulation period) is $\beta$ independent and is a decreasing function of $N_{c}$ down to $\tau_{l} / \tau_{c}\left(N_{c} \geqslant 1.4\right) \approx 1$. The ratio of the normalized speckle displacements $\eta \equiv\left(\delta \mathrm{x} / w_{\mathrm{sp}}\right) /\left(\delta \mathrm{z} / l_{\mathrm{sp}}\right)$ is larger than 1 , for $N_{c}$ $<1.25$ and $1.5<N_{c}<2.3$, which means that during its motion one speckle crosses more speckle widths than it crosses speckle lengths. As the ratio of the displacement times $\left(w_{\mathrm{sp}} / v_{\perp}\right) /\left(l_{\mathrm{sp}} / v_{\|}\right)$is simply $\eta^{-1}$, we can claim that, within the same ranges, the coherence time is reduced more by the tranverse motion than by the axial motion.

When propagating in the plasma, speckles can self-focus by digging density depressions. In isolation, one immobile speckle leads to a steady-state cavity with amplitude $\delta n_{s 0} / n_{0}=-2.4 \times 10^{-4} I_{14}$ (in our conditions where linear hydrodynamics is fully valid) with $I_{14}$ the irradiance in $10^{14} \mathrm{~W} / \mathrm{cm}^{2}$. The plasma is expelled over time $\tau_{\mathrm{exp}} \equiv w_{\mathrm{sp}} / c_{s}$ $=4.3 \mathrm{ps}$ and then brought away via density bumps with $w_{\mathrm{sp}}$ width at velocity $c_{s}$. If the injected speckles have no motion, the transmitted light does not exhibit any speckle velocity. By contrast, oscillating speckles induce a speckle motion in the transmitted light, whose aspect over $350 \mu \mathrm{m}$ propagation shows either a steepening or a flattening of the initial velocity profile (Fig. 2), depending on the quality of the beam smoothing. We have measured the fraction of the beam energy with local irradiance above 5 times the average irradiance, ${ }^{7}$ from which we have defined the time average $P_{5}(z)$. The Gaussian statistics in the focal $\operatorname{spot}^{15}$ lead to: $P_{n=5}(0)=(n+1) e^{-n}=4 \%$. In a typical case of weak smoothing [Fig. 2(a)], $P_{5}(z)$ increases up to $18 \%$ before de- 

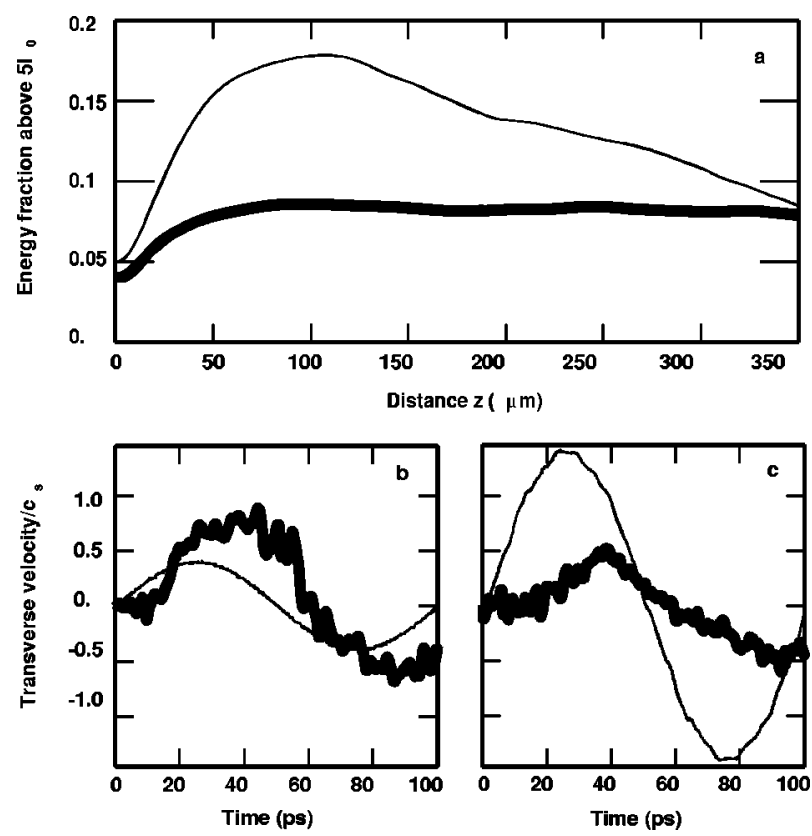

FIG. 2. (a) Fraction of energy above five times the average irradiance, as a function of the propagation distance for $N_{c}=0.4, \beta=1$ (thin line), and $N_{c}$ $=1, \beta=4$ (thick line). Transverse speckle velocity as a function of time, for the incident light $(z=0$ : thin line $)$ and the transmitted light $(z=350 \mu \mathrm{m}$ : thick line), for (b) $N_{c}=0.4, \beta=1$, and (c) $N_{c}=1, \beta=4$. The laser irradiance is: $I_{0}=2 \times 10^{15} \mathrm{~W} / \mathrm{cm}^{2}$ with $\nu_{m}=10 \mathrm{GHz}$ and the plasma conditions are: $n_{e 0} / n_{c}=0.1, T_{e}=3 \mathrm{keV}, \nu_{s} / \omega_{s}=0.1$.

creasing beyond a $100 \mu \mathrm{m}$ distance, whereas, in a case of efficient smoothing, $P_{5}(z)$ reaches only $7 \%$ and remains nearly constant beyond a $60 \mu \mathrm{m}$ propagation distance. The scenario of velocity profile steepening [Fig. 2(b)] is associated with weak beam smoothing, and, as a result, with selffocusing: Each intense speckle propagates in its own channel, ignoring its neighbors. The speckles bend in the direction opposite to the speckle velocity (as if a fixed speckle was injected in a plasma drifting along the opposite direction), with a sudden deviation (increasing with the speckle intensity) at the point where the self-focusing is maximum (and the plasma the more dug). As the speckle position oscillates with time, the speckle trajectory bends in both positive and negative $x$ directions like a long whip: As a result, the velocity of the transmitted light varies strongly when the speckle has reached its extremal position and shows a steepened time variation. This scenario has been recovered by propagating one isolated Gaussian speckle (4 $\mu \mathrm{m}$ width, $6 \times 10^{15} \mathrm{~W} / \mathrm{cm}^{2}$ peak irradiance, 1.65 times the critical power for self-focusing). The injected sinusoidal velocity profile steepened after $140 \mu \mathrm{m}$ propagation, due to the filament bending, and showed strong oscillations and an increase in the peak velocity for further propagation, due to the reversed filament motion. The other scenario is shown in Fig. 2 (c), where the speckles are seen to slow down by propagating in the plasma. The beam smoothing is efficient enough to satisfy $\tau_{l}=\tau_{c}<\tau_{\text {exp }}$. The channel dug by a speckle cannot be sustained long enough to trigger strong self-focusing and consequently the speckle experiences multiple scattering off both the plasma bumps and holes (when not sustained by a permanent $\mathrm{PF}$, the hole relaxes as a ring-shaped cavity ex-

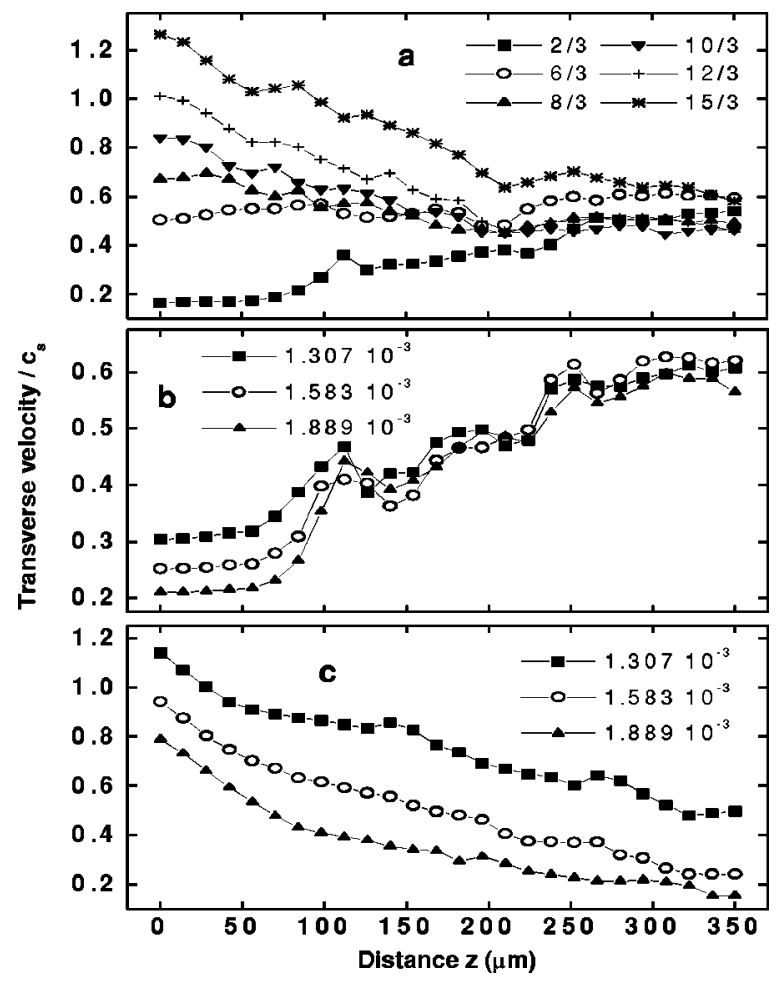

FIG. 3. Transverse speckle velocity as a function of the propagation distance for (a) different values of $\beta$ and $N_{c}=0.4$ (see other conditions in Fig. 2 caption), (b) $\beta=1, N_{c}=0.4$ for different values of $c_{s} / c$ [intensity $I_{0}$ and electron temperature $T_{e}$ are varied so that $I_{0} /\left(T_{e}+3 T_{i} / Z\right)$ is kept constant] and (c) $\beta=4, N_{c}=1$ and same conditions as for (b).

panding at velocity $c_{s}$ ). The resulting transmitted light does not preserve the incident time history; the longer the plasma, the more random the speckle trajectory and the smaller the average speckle velocity.

For $N_{c}=0.4$ (weak smoothing), Fig. 3(a) shows how the speckle velocity (time average of the positive velocities denoted $M c_{s}$ ) depends on the plasma length and the modulation depth $\beta$. Whereas, in the focal spot, $M$ increases with $\beta$ in the range $[0.15,1.25]$, the velocities in the transmitted light do not show such ordering and are located in the smaller range $[0.44,0.6]$, exhibiting some asymptotic velocity. Decreasing the IAW damping boosts the self-focusing process and makes the distance for asymptotic velocity decrease: 100 (respectively, 160) $\mu \mathrm{m}$, for $\nu_{s} / \omega_{s}=0.025$ (respectively, 0.05) [the velocity ranges become $[0.44,0.51]$ (respectively $[0.38,0.54])]$. In order to gauge the influence of the IAW velocity, the ion and electron temperatures and the irradiance have been varied, while keeping constant the ratio $I_{0} /\left(T_{e}+3 T_{i} / Z\right)$ and thus the steady-state density depression $\delta n_{s}$. For $c_{s} / c=1.3,1.58$, and $1.89 \times 10^{-3}$, the transmitted speckle velocity has been measured to be $M=0.60,0.62$ and 0.58 , respectively [Fig. 3(b)]. These results are very close to $(2 / \pi)$, which would be the average velocity of one halfperiod of a sinusoidal oscillation with $c_{s}$ as the maximum velocity. Whatever the maximum speckle velocity $v_{M}$ is, the speckle velocities approach the sonic velocity. If $M_{M}$ $\equiv v_{M} / c_{s}<1$, the steady-state plasma depression becomes $\delta n_{0} / \sqrt{1-M_{M}^{2}} 16$ and the self-focusing is enhanced twice each period when the velocity varies gently around $\pm v_{M}$, 


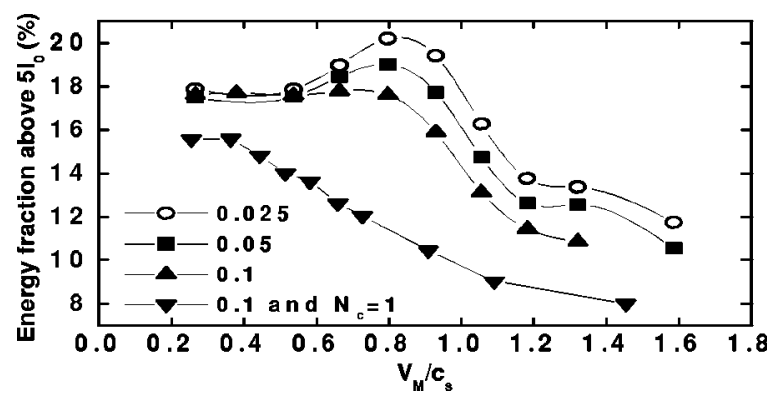

FIG. 4. Fraction of energy above five times the average irradiance, as a function of the maximal speckle velocity in the focal spot, for $N_{c}=0.4$ and 1 , and different values of $\nu_{s} / \omega_{s}$.

which then increases the speckle velocity of the transmitted light. For $M_{M}>1$, the maximum velocity does not induce strong self-focusing and only for times where the instantaneous speckle velocity is subsonic and close to $c_{s}$ does selffocusing occur. But as the velocity varies rapidly at those times, the depression cannot be stabilized and the deviation is smaller than in the case $M_{M}<1$. As a result, the speckle velocity in the transmitted light is smaller than what it is in the focal spot. This explains qualitatively the existence of this asymptotic velocity; a more quantitative prediction would require further investigation. For larger bandwidth $\left(N_{c}=1, \beta=4\right)$, i.e., efficient beam smoothing, the speckle velocity decreases by propagating, more rapidly at higher temperature [Fig. 3(c)]. This exhibits two nonlinear regimes for self-focusing and beam bending: (i) a weakly nonlinear regime, illustrated by Figs. 2(c) and 3(c), for which selffocusing is partly prevented by efficient beam smoothing and the initial speckle velocity reduces dramatically by propagation, (ii) a fully nonlinear regime, illustrated by Figs. 2(b), $3(\mathrm{a})$, and 3(b), exhibiting self-focusing and an asymptotic velocity close to $c_{s}$.

We have estimated the beam smoothing quality by varying the laser bandwidth. Clearly, the latter modifies the filamentation strength but not according to a monotonic law (Fig. 4). By increasing $\beta$, the speckle velocity is increased and can become supersonic $\left(M_{M}>1\right)$. In this case, whatever the IAW damping may be, the fraction of energy $P_{5}$ (maximum over $z$ ) decreases substantially as $M_{M}$ is increased. For $\nu_{s} / \omega_{s}=0.1, P_{5}$ is constant in the subsonic region and does not exhibit enhancement when approaching the sonic point. By contrast, for $\nu_{s} / \omega_{s}=0.025$ (low ion temperature), $P_{5}$ is in excess of $20 \%$ for $M_{M}=0.8$. The resonance at this subsonic velocity results from a compromise between the larger difference between peak and valley density values induced by a speckle velocity approaching $c_{s}$ and the finite speckle lifetime which decreases with increasing $\beta$. The difference between peak and valley density is shown to be much more important for the low damping case than for the standard one (Fig. 5).

In conclusion, the plasma nonlinearity clearly modifies the average speckle velocity induced by the 1-D transverse

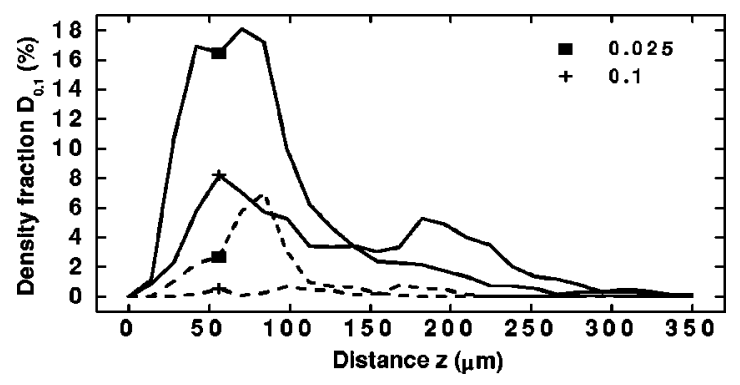

FIG. 5. Fraction of density perturbations $10 \%$ above (dotted line) and below (solid line) the equilibrium density value. See other conditions in Fig. 2 caption.

SSD. A resonance between the speckle motion and plasma dynamics effect has been shown for insufficient beam smoothing and for small IAW damping, whenever the peak speckle velocity is close to the IAW velocity. This effect is not observed in the context of megajoule laser optical design, with a cycle number of 1 and large ion damping, because the speckle lifetime is too short. The control of the speckle velocity appears not to be a constraint but a comparison or combination with other kinds of smoothing techniques requires further investigation. Also, further study is required to consider plasma drifting and to reconsider our observations as a function of the drift velocity as a new parameter.

\section{ACKNOWLEDGMENTS}

Discussions with R. Berger, J. Garnier, C. Gouedard, and L. Videau are gratefully acknowledged.

${ }^{1}$ B. Mc Gowan, B. B. Afeyan, C. A. Back et al., Phys. Plasmas 3, 2029 (1996); J. C. Fernandez, J. A. Cobble, B. H. Failor et al., Phys. Rev. Lett. 77, 2702 (1996); D. S. Montgomery, B. B. Afeyan, J. A. Cobble et al., Phys. Plasmas 5, 1973 (1998).

${ }^{2}$ S. Haan, S. M. Pollaine, J. D. Lindl et al., Phys. Plasmas 2, 2480 (1995); A. Jolas, C. Bayer, E. Dattolo et al., in Inertial Fusion Sciences and Applications, edited by C. Labaune, W. J. Hogan, and K. A. Tanaka (Elsevier, Paris, 2000), pp. 68-73.

${ }^{3}$ J. D. Moody, B. J. MacGowan, D. E. Hinkel et al., Phys. Rev. Lett. 77, 1294 (1996).

${ }^{4}$ S. Skupsky, R. W. Short, T. Kessler et al., J. Appl. Phys. 66, 3456 (1989).

${ }^{5}$ C. E. Max, Phys. Fluids 19, 74 (1976); P. Kaw, G. Schmidt, and T. Wilcox, ibid. 16, 1522 (1973).

${ }^{6}$ A. J. Schmitt, Phys. Fluids 31, 3079 (1988).

${ }^{7}$ R. L. Berger, B. Lasinski, T. Kaiser, E. A. Williams, and B. Langdon, Phys. Fluids B 5, 2243 (1993).

${ }^{8}$ D. Montgomery, J. Moody, H. Baldis, B. Afeyan, R. L. Berger, K. Estabrook, B. Lasinski, and E. Williams, Phys. Plasmas 3, 1728 (1996).

${ }^{9}$ A. J. Schmitt and B. B. Afeyan, Phys. Plasmas 5, 503 (1998).

${ }^{10}$ G. Riazuelo and G. Bonnaud, Phys. Plasmas 7, 3841 (2000).

${ }^{11}$ A. V. Maximov, I. G. Ourdev, D. Pesme et al. Phys. Plasmas 8, 1319 (2001).

${ }^{12}$ M. D. Feit and J. A. Fleck, J. Opt. Soc. Am. B 5, 633 (1988); G. Riazuelo, Ph.D. thesis (in French), Paris XI University, 1999.

${ }^{13}$ The maximum of the function $C$ has been calculated by precisely locating the maximum, via a dichotomy method; a two-mesh interval has been used along every direction around a first indication of the mesh where $C$ maximizes, with use of the seventh-order Lagrange interpolation function.

${ }^{14}$ L. Videau, C. Rouyer, J. Garnier, and A. Migus, J. Opt. Soc. Am. A 16, 1672 (1999).

${ }^{15}$ J. Garnier, Phys. Plasmas 6, 1601 (1999).

${ }^{16}$ H. Rose, Phys. Plasmas 3, 1709 (1996); D. E. Hinkel, E. A. Williams, and C. H. Still, Phys. Rev. Lett. 77, 1298 (1996). 